\title{
ECONOMIC GROWTH AND HEALTH DYNAMICS WITH GOVERNMENT SUBSIDIES FOR HEALTHCARE
}

\section{Wei-Bin Zhang*}

\begin{abstract}
The aim of this paper is to examine the dynamic interdependence between wealth accumulation and health change with government health policy. The paper focuses on how government health policy affects health change, economic growth and the dynamics of government debt within a dynamic general equilibrium framework proposed by Zhang. Economic growth, health change and government debt dynamics are interrelated with different preferences and government health policy. After building the general equilibrium model, it is demonstrated that the movement of the economic system is provided by three nonlinear differential equations. We simulate the differential equations and show how the economic system moves from its initial state. A comparative dynamic analysis is conducted in order to examine how changes in some exogenous conditions affect short-run transitory processes and the equilibrium structure.
\end{abstract}

Keywords: health dynamics; economic growth; government debt; government subsidy; system stability

JEL Classification: H51, I15, O41

\section{Introduction}

One's health condition is generally considered to be a significant determinant of economic growth in literature addressing growth and health (e.g., Grossman, 1972; Ehrlich and Chuma, 1990; Acemoglu and Johnson, 2007; Chen et al., 2014; Zhang, 2018). A healthy labour force tends to be highly productive. On the other hand, a productive economy can also provide resources for healthcare. Moreover, it is obvious that governments can play an important role in improving the population's health by providing resources for healthcare. There are also issues related to government debts and financial support for healthcare. There are some studies on the impact from health on national and household economic conditions (e.g., Parkin et al., 1987; Posnett and Hitiris, 1992; Strauss and Thomas, 1998; Schultz, 1999; Bloom, et al., 2004; Eggoh et al. 2015; Jel Bedir, 2016). Some studies in literature find positive effects of health on economic growth (e.g., Bloom and Canning, 2003). There are also studies on relationships between healthcare expenditures and income and the magnitude of income elasticity of expenditures (Newhouse, 1977; Yavuz et al., 2011; Baltagi and Moscone, 2010; Murray et al., 1994). Our approach is influenced by the extensive literature. This study differs from most of the literature in that it addresses the dynamic interactions among many

* Ritsumeikan Asia Pacific University, Japan (wbz1@apu.ac.jp). The author is grateful for important and constructive comments of the two anonymous referees. 
variables in a general equilibrium framework on a microeconomic foundation by integrating some well-known models into economic theory.

As interactions between growth and health involve dynamic analysis, it is challenging to build dynamic relations on a microeconomic foundation. It is difficult to analyse the properties of nonlinear dynamic economic systems with many complicated interactions. Although there are some theoretical studies about dynamic interactions between growth and health, there are only a few theoretical models that explicitly take account of government debts in literature on growth and health. We built our model on the basis of the neoclassical growth theory and the Diamond growth model with debt (Diamond, 1965). Government debt is an important issue in modern economies. The dynamics of government debts are complicated as debts have many causes that affects these factors. Debts have complicated relations with GDP, taxation structures, population structure and economic growth. We study the dynamics of debts with government health policy and different taxes in a competitive economy. In our approach, wealth and health are the engines of economic growth. Neoclassical growth theory treats wealth accumulation as the engine of growth (Solow, 2000; Burmeister and Dobell, 1970; and Zhang, 2005). We follow this tradition and add health dynamics following the approach by Grossman (1972). In summary, our model integrates three well-known models - Solow's growth, Diamond's debt, and Grossman's health - in a comprehensive framework by applying Zhang's concept of disposable income and utility function. It should be mentioned that the model in this paper is also based on Zhang's two models. Zhang (2016) built a two-sector growth model on the basis of Diamond's wellknown growth model with debt. Zhang (2018) introduced endogenous health accumulation and a healthcare sector into the Solow one-sector growth model. The rest of the paper is organised as follows. Section 1 develops the two-sector model with wealth accumulation and health change with government subsidy on health and government debt; Section 2 provides a computational procedure and simulates the model. The simulation plots the movement of the system for a short period of time and identifies an unstable equilibrium point; Section 3 conducts a comparative dynamic analysis in different parameters to show how the paths of economic development are affected by exogenous changes in the environment.

\section{The Basic Model}

We are now building a two-sector growth with wealth and health. The economy consists of industrial and health sectors. Most aspects of the two sectors are similar to the multisector growth models in neoclassical growth theory (Burmeister and Dobell, 1970 and Zhang, 2005). The industrial sector produces homogeneous durable goods, which is used for consumption and investment. The health sector supplies healthcare services. Households own assets of the economy and distribute their incomes to consume and save. All markets are perfectly competitive. Input factors are fully employed by the two sectors. There is a homogenous and fixed population. We select commodity to serve as numeraire and all prices are measured relative to its price. Perfect competition and free mobility of input factors imply equalisation of the interest rate and the wage rate between the two sectors. We introduce the following variables:

$\bar{N}$ and $N(t)$ - fixed population and labour force at time $t$;

$i$ and $\ell$-subscript index standing for industrial and health sectors, respectively;

$K(t)$ - total capital stock; 
$F_{j}(t)$ - output of sector $j, j=i, \ell$;

$N_{j}(t)$ and $K_{j}(t)$ - labour force and capital stock employed by sector $j, j=i, \ell$;

$r(t)$ and $w(t)$ - rate of interest and wage rate per unit of time;

$T(t), \bar{T}(t), T_{\ell}(t)$ - work hour, leisure time, and time spent on healthcare;

$D(t)$ - government debt;

$c(t)$ and $s(t)$ - consumption and saving of the representative household;

$a(t)$ - value of wealth owned by the representative household;

$\tau_{j}$ and $\bar{\tau}_{j}-$ government tax rate on sector $j^{\prime} s$ output and $\bar{\tau}_{j} \equiv 1-\tau_{j}$;

$\tau_{a}$ and $\bar{\tau}_{a}$-government tax rate on household wealth income and $\bar{\tau}_{a} \equiv 1-\tau_{a}$;

$\tau_{w}$ and $\bar{\tau}_{w}-$ government tax rate on household wage income and $\bar{\tau}_{w} \equiv 1-\tau_{w}$;

$\tau_{c}$ and $\tilde{\tau}_{c}-$ government tax rate on household consumption and $\tilde{\tau}_{c} \equiv 1+\tau_{c}$;

$\delta_{k}$ and $\delta_{\ell}-$ depreciation rates of physical capital and health.

The total labour force is specified as follows:

$$
N(t)=h T(t) \ell^{m_{\ell}}(t) \bar{N}
$$

in which $m_{\ell}$ measures how health affects a worker's productivity and $h$ is human capital. This formation of the labour force is frequently used in literature (e.g., Well, 2007; Tobing, 2011; and Zhang 2018).

\section{The two sectors}

The two sectors' technologies are described by the following conventional production functions:

$$
F_{j}(t)=A_{j} K_{j}^{\alpha_{j}}(t) N_{j}^{\beta_{j}}(t), A_{j}, \alpha_{j}, \beta_{j}>0, \alpha_{j}+\beta_{j}=1, j=i, \ell,
$$

where $A_{j}, \alpha_{j}$, and $\beta_{j}$ are parameters. Markets are competitive; thus labour and capital earn their marginal products. The Cobb-Douglas production functions and perfect competition imply that firms have zero profits. We use $p_{j}(t)$ to stand for the price of sector $j$, with $p_{i}(t)=1$. Sector $j$ decides $K_{j}(t)$ and $N_{j}(t)$ to maximise the profit. The marginal conditions imply:

$$
r(t)+\delta_{k}=\frac{\alpha_{j} \bar{\tau}_{j} p_{j}(t) F_{j}(t)}{K_{j}(t)}, w(t)=\frac{\beta_{j} \bar{\tau}_{j} p_{j}(t) F_{j}(t)}{N_{j}(t)} .
$$

\section{Quality of healthcare}

We follow Grossman (1972) in modelling healthcare. We consider that the output of healthcare, $\bar{c}(t)$, is related to the level of the health service purchased on the market and the time spent on healthcare. As in Zhang (2018), the quality of healthcare is dependent on $c_{\ell}(t)$ and $T_{\ell}(t)$ as follows:

$$
\bar{c}(t)=\bar{A} c_{\ell}^{\bar{\alpha}}(t) T_{\ell}^{\bar{\beta}}(t), \bar{A}, \bar{\alpha}, \bar{\beta}>0,
$$

where $\bar{A}, \bar{\alpha}$, and $\bar{\beta}$ are the parameters. 


\section{Current income and disposable income}

This study applies Zhang's approach to household behaviour (Zhang, 1993; 2005). Zhang proposed an alternative utility function and the concept of disposable income to replace the fixed saving rate approach without microeconomic foundation as in the Solow growth model and the infinitely discounted utility approach in the Ramsey growth theory. We use $\bar{k}(t)$ to stand for physical wealth per household. We have $\bar{k}(t)=K(t) / \bar{N}$. The household wealth is the sum of the physical wealth and government debt as follows:

$$
a(t)=\bar{k}(t)+d(t)
$$

where $d(t)$ is the debt owned by the representative household. We have $d(t)=D(t) / \bar{N}$. The current income from the interest payment and the wage payment is

$$
y(t)=\bar{\tau}_{a}(t) r(t) a(t)+\tilde{w}(t) T(t),
$$

where

$$
\tilde{w}(t) \equiv \bar{\tau}_{w} \bar{\ell}(t) w(t), \quad \bar{\ell}(t) \equiv h \ell^{m_{\ell}}(t) .
$$

It should be remarked that the current income in Zhang's approach is the disposable income in the Solow growth model. We assume that selling and buying physical wealth and debt is conducted instantaneously without any transaction cost. The total value of wealth that the household can sell to purchase goods and to save is equal to $a(t)$. The disposable income in Zhang's approach is the sum of the current income and the value of the household wealth is as follows:

$$
\hat{y}(t)=y(t)+a(t) .
$$

The disposable income is used up between saving, consumption, and healthcare.

\section{The budget, utility function, and optimal behaviour}

Let $\hat{\tau}(t)$ stand for the subsidy per unit of health people receive from the government. The healthcare cost $\bar{p}_{\ell}(t)$ is the difference between the price of healthcare and the subsidy rate:

$$
\bar{p}_{\ell}(t)=p_{\ell}(t)-\hat{\tau}(t)
$$

The household's budget constraint implies:

$$
\bar{\tau}_{c} c(t)+s(t)+\bar{p}_{\ell}(t) c_{\ell}(t)=\hat{y}(t)
$$

Each household is subject to the following time constraint:

$$
T(t)+\bar{T}(t)+T_{\ell}(t)=T_{0},
$$


where $T_{0}$ represents the available time for work, leisure and healthcare. Insert (9) in (8)

$$
\hat{y}(t)=\bar{y}(t)-\tilde{w}(t) T_{\ell}(t)-\tilde{w}(t) \bar{T}(t),
$$

where

$$
\bar{y}(t)=\tilde{R}(t) a(t)+\tilde{w}(t) T_{0}, \quad \tilde{R}(t) \equiv 1+\bar{\tau}_{a}(t) r(t) .
$$

Insert (10) in (8)

$$
\tilde{w}(t) T_{\ell}(t)+\tilde{w}(t) \bar{T}(t)+\bar{\tau}_{c} c(t)+s(t)+\bar{p}_{\ell}(t) c_{\ell}(t)=\bar{y}(t) .
$$

The utility function is a function of $c(t), s(t), \bar{c}_{\ell}(t)$, and $\bar{T}(t)$ as follows:

$$
U(t)=c^{\xi_{0}}(t) s^{\lambda_{0}}(t) \bar{c}^{\psi_{0}}(t) \bar{T}^{\sigma_{0}}(t), \xi_{0}, \lambda_{0}, \psi_{0}, \sigma_{0}>0,
$$

where $\xi_{0}$ is called the propensity to consume, $\sigma_{0}$ the propensity to use leisure, $\psi_{0}$ the propensity to use health service, and $\lambda_{0}$ the propensity to own wealth and. Insert (4) in (12)

$$
U(t)=\bar{A}^{\psi_{0}} c^{\xi_{0}}(t) s^{\lambda_{0}}(t) c_{\ell}^{\bar{\alpha} \psi_{0}}(t) T_{\ell}^{\bar{\beta} \psi_{0}}(t) \bar{T}^{\sigma_{0}}(t)
$$

Maximizing the above $U(t)$ subject to (8), we have the following marginal conditions:

$$
\begin{gathered}
c(t)=\xi \bar{y}(t), \quad s(t)=\lambda \bar{y}(t), \quad \bar{p}_{\ell}(t) c_{\ell}(t)=\psi_{\ell} \bar{y}(t), \quad \tilde{w}(t) T_{\ell}(t)=\psi_{T} \bar{y}(t), \\
\tilde{w}(t) \bar{T}(t)=\sigma \bar{y}(t),
\end{gathered}
$$

where

$$
\begin{aligned}
& \xi \equiv \frac{\rho \xi_{0}}{\bar{\tau}_{c}}, \lambda \equiv \rho \lambda_{0}, \quad \psi_{\ell} \equiv \rho \bar{\alpha} \psi_{0}, \\
& \psi_{T} \equiv \rho \bar{\beta} \psi_{0}, \quad \sigma \equiv \rho \sigma_{0}, \quad \rho=\frac{1}{\xi_{0}+\lambda_{0}+\bar{\alpha} \psi_{0}+\bar{\beta} \psi_{0}+\sigma_{0}} .
\end{aligned}
$$

\section{Wealth accumulation}

The change in the household's wealth is savings minus dissavings as follows:

$$
\dot{a}(t)=s(t)-a(t) .
$$




\section{Government expenditure, tax income, and debt}

This study assumes that each household receives a health subsidy in proportion to the price as follows:

$$
\hat{\tau}(t)=\tau_{0} p_{\ell}(t),
$$

where ( 1) is a non-negative parameter. This implies that the government subsidy is endogenously determined in such a way that the price that each student actually pays per unit of time is $(1-\hat{\tau}) p_{\ell}(t)$, that is, $\bar{p}_{\ell}(t)=\left(1-\tau_{0}\right) p_{\ell}(t)$.

The government income comes from collecting taxes and issuing interest-bearing debt. We use $I_{T}(t)$ to represent the government income. The income is the tax incomes from the two sectors, interest income of wealth, and consumption as follows:

$$
I_{T}(t)=\tau_{i} F_{i}(t)+\tau_{\ell} p_{\ell}(t) F_{\ell}(t)+\tau_{a} r(t) a(t) \bar{N}+\tau_{c} c(t) \bar{N}+\tau_{w} \bar{\ell}(t) T(t) w(t) \bar{N} .
$$

It should be remarked that in the Diamond model and other dynamic models with debts, the tax rates are fixed. Since the government spends only on healthcare, its expenditure is as follows:

$$
T_{p}(t)=\hat{\tau} p_{\ell}(t) \bar{N}
$$

The government debt follows the following dynamics:

$$
\dot{D}(t)=r(t) D(t)+\tau_{0} p_{\ell}(t) \bar{N}-T_{p}(t) .
$$

\section{Market equilibrium conditions}

Demand for and supply of supply balances

$$
c_{\ell}(t) \bar{N}=F_{\ell}(t) .
$$

As the output of the industrial sector is equal to the sum of the level of consumption, the depreciation of capital stock and the net savings, we have

$$
C(t)+S(t)-K(t)+\delta_{k} K(t)=F_{i}(t),
$$

where

$$
C(t)=c(t) \bar{N}, \quad S(t)=s(t) \bar{N} .
$$




\section{Health dynamics}

As in Zhang (2018), the change in health stock is dependent on nutrition and healthcare. We propose the health dynamics as follows:

$$
\dot{\ell}(t)=\frac{\bar{v}_{\ell} \bar{c}^{\bar{a}_{\ell}}(t)}{\ell^{\bar{\pi}_{\ell}}(t)}+\frac{\bar{v}_{c} c^{\bar{a}_{c}}(t)}{\ell^{\bar{c}_{c}}(t)}+\frac{\bar{v}_{T} \bar{T}^{\bar{a}_{T}}(t)}{\ell^{\bar{\pi}_{T}}(t)}-\delta_{\ell} \ell(t),
$$

where $\bar{\delta}_{h}(>0)$ is the depreciation rate of health stock, $\bar{v}_{\ell}, \bar{v}_{c}, \bar{v}_{T}, \bar{a}_{\ell}, \bar{a}_{c}$ and $\bar{a}_{T}$ are non-negative parameters (see also Grossman, 1972; Tobing, 2011; Johansson and Löfgren, 1995). The signs of the returns-to-scale parameters $\bar{\pi}_{\ell}, \bar{\pi}_{c}$ and $\bar{\pi}_{T}$ are not specified as they may be either negative or positive. The term $\bar{v}_{\ell} \bar{c}^{\bar{a}_{\ell}} / \ell^{\bar{\pi}_{\ell}}$ means that the improvement in health is positively related to the investment in healthcare and there are returns to scale effect in health improvement. We can similarly interpret $\bar{v}_{c} c^{\bar{a}_{c}} / \ell^{\bar{\pi}_{c}}$ and $\bar{v}_{T} \bar{T}^{\bar{a}_{T}} / \ell^{\bar{\pi}_{T}}$.

\section{Full employment of input factors}

We have

$$
N_{i}(t)+N_{\ell}(t)=N(t), \quad K_{i}(t)+K_{\ell}(t)=K(t) .
$$

All the physical capital is owned by households

$$
\bar{k}(t) \bar{N}=K(t) .
$$

Therefore, we built the dynamic model, which is an integration of Solow's onesector neoclassical growth model, Diamond's growth model with government debt, and Grossman's growth model with endogenous health. We study the dynamic properties of the economic system in the following section.

\section{The Dynamics and Properties}

We study the dynamic behaviour of the model. The following lemma gives a computational procedure for us to follow the movement of the economic dynamics. Before stating the lemma, we introduce a variable as follows:

$$
z(t) \equiv \frac{r(t)+\delta_{k}}{w(t)} .
$$

\section{Lemma}

The following three differential equations determine the movement of the three variables, $z(t), D(t)$ and $\ell(t)$ :

$$
\begin{gathered}
\dot{z}(t)=\Lambda_{z}(z(t), D(t), \ell(t)), \\
\dot{D}(t)=\Lambda_{D}(z(t), D(t), \ell(t)), \\
\dot{\ell}(t)=\Lambda_{\ell}(z(t), D(t), \ell(t)) .
\end{gathered}
$$


The three functions, $\Omega_{z}(t), \Omega_{H}(t)$, and $\Omega_{\ell}(t)$ are defined in the appendix. Moreover, the other variables are determined as functions of $z(t), D(t)$ and $\ell(t)$ by the following procedure: $r(t)$ by (A2) $\rightarrow w(t)$ with (A3) $\rightarrow p_{\ell}(t)$ by (A4) $\rightarrow a(t)$ with (A16) $\rightarrow \bar{y}(t)$ from $(\mathrm{A} 5) \rightarrow d(t)=D(t) / \bar{N} \rightarrow \bar{k}(t)=a(t)-d(t) \rightarrow s(t), \hat{T}(t), \bar{T}(t), c(t)$, and $c_{\ell}(t)$ by (13) $\rightarrow T(t)$ with (9) $\rightarrow N(t)$ by (1) $\rightarrow N_{\ell}(t)$ from (A9) $\rightarrow N_{i}(t)=N(t)-N_{\ell}(t) \rightarrow$ $K_{i}(t), K_{\ell}(t)$ by (A1) $\rightarrow K(t)$ by (22) $\rightarrow F_{q}(t)$ by (2) $\rightarrow \bar{c}(t)$ from (4) $\rightarrow T_{p}(t)$ by (A13).

Instead of the wealth, we use the cost ratio of the two input factors as a variable of the dynamics. As shown in the appendix, this new variable enables us to explicitly find the differential equations for simulation. With the lemma, we can follow the movement of the system over time. As we cannot provide an explicit system solution, we simulate the model. We specify $T_{0}=24$ and the population $\bar{N}=10$. We specify the parameter values as follows:

$$
\begin{aligned}
& h=2, \tau_{0}=0.6, m_{\ell}=0.6, \quad \alpha_{i}=0.33, \quad \alpha_{\ell}=0.4, \quad \bar{\alpha}=0.4, \quad \bar{\beta}=0.38, \quad A_{i}=1.3, \\
& A_{\ell}=1, \bar{A}=1, \quad \lambda_{0}=0.7, \quad \xi_{0}=0.1, \quad \psi_{0}=0.04, \quad \sigma_{0}=0.2, \quad \delta_{k}=0.04, \quad \delta_{\ell}=0.04 .
\end{aligned}
$$

The government subsidises $60 \%$ of the cost of healthcare. The values of $\alpha_{x}$ in the Cobb-Douglas productions are closely equal to 0.3 . These parameter values are used to estimate production functions (see Miles and Scott, 2005; Abel et al., 2007). The total factor productivities of the two sectors are $A_{i}=1.3$ and $A_{\ell}$. Depreciation rates of physical capital are often approximately fixed at 0.05 in empirical studies. We specify $\bar{\alpha}=0.4$ and $\bar{\beta}=0.38$, which means decreasing returns in healthcare (e.g., Forster, 1989; Ehrlich and Chuma, 1990; van Zon and Muysken, 2001). The propensity to save is 0.7, which implies that about $70 \%$ of the disposable income is used for savings. We specify the parameters in the change of health stock and tax rates as follows:

$$
\begin{aligned}
& \bar{v}_{\ell}=0.7, \quad \bar{v}_{c}=0.5, \quad \bar{v}_{T}=0.7, \quad \bar{\pi}_{\ell}=0.4, \bar{\pi}_{c}=0.7, \quad \bar{\pi}_{T}=0.8, \quad \bar{a}_{\ell}=0.4, \\
& \bar{a}_{c}=0.2, \quad \bar{a}_{T}=0.2, \quad \tau_{\ell}=0.05, \tau_{i}=0.04, \tau_{a}=0.1, \tau_{c}=0.02, \tau_{w}=0.02 .
\end{aligned}
$$

We choose $\bar{\pi}_{\ell}=0.4, \bar{\pi}_{i}=0.4$, and $\pi_{T}=0.8$ to imply that the health stock change exhibits decreasing returns in the three factors. We specify the initial conditions as follows:

$$
z(0)=0.054, \quad D(0)=3590, \ell(0)=18.2 .
$$

The simulation result is plotted in Figure 1. The system does not show any convergence towards an equilibrium point. 


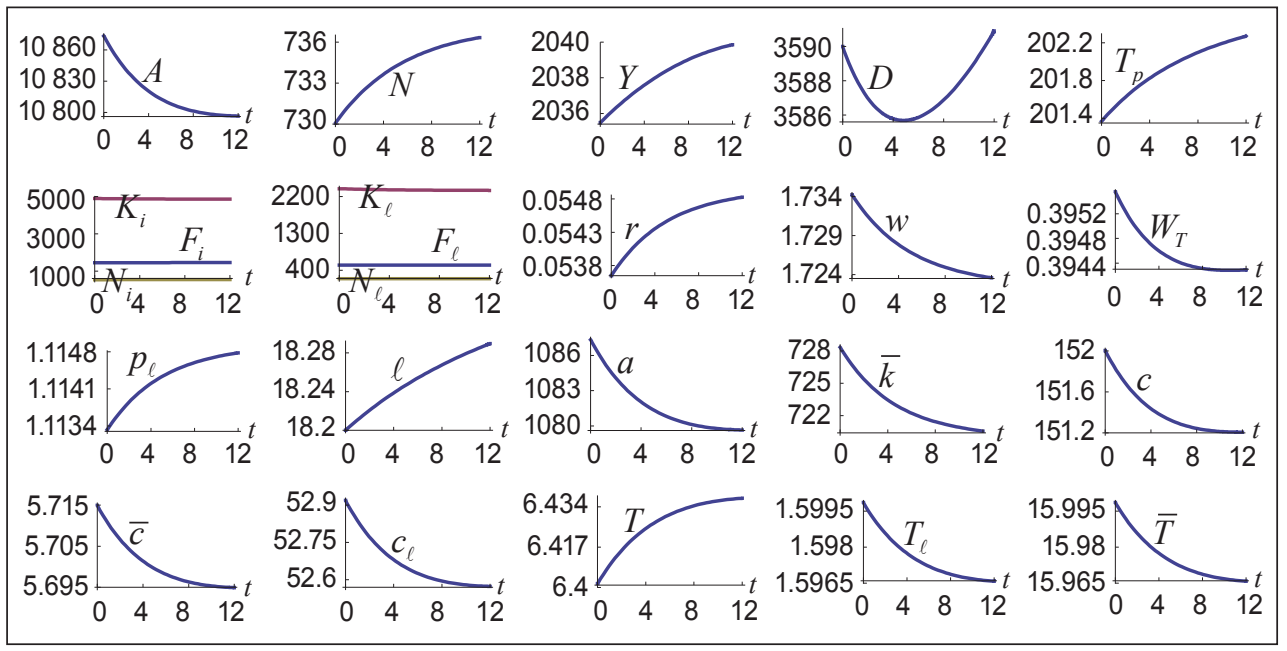

Source: author

The simulation demonstrates that the dynamic system has two equilibrium points. We list the values of one equilibrium point as follows:

$$
\begin{aligned}
& Y=2048.2, \quad T_{p}=202.9, \quad \bar{K}=7243.1, \quad A=10828.8, \quad N=738.9, \quad D=3585.7, \\
& K_{i}=4884.6, \quad K_{\ell}=2358.5, \quad N_{i}=544.7, \quad N_{\ell}=194.3, \quad F_{i}=1460.4, \quad F_{\ell}=527.4, \\
& r=0.055, \quad w=1.73, \quad W_{T}=0.4, \quad p_{\ell}=1.12, \quad \ell=18.4, \quad a=1082.9, \quad \bar{k}=724.3, \\
& c=151.7, \quad c_{\ell}=52.7, \quad \bar{c}=5.7, \quad T=6.4, \quad T_{\ell}=1.6, \quad \bar{T}=17 .
\end{aligned}
$$

The equilibrium point is near to the initial state as specified in Figure 1. We calculate the three eigenvalues as follows:

$$
-0.193,0.085,-0.06
$$

As one eigenvalue is real and positive, the equilibrium is unstable. The system does not approach this equilibrium.

By simulation, we identify the other equilibrium point as follows:

$$
z=0.011, \quad D=-72634, \quad \ell=19.03 \text {. }
$$

We are not concerned with this equilibrium point, partly because the government debt is largely negative. The rest of the study is concerned with the movement of the system near the paths given in Figure 1. 


\section{Short-Run Comparative Dynamic Analysis}

This section is concerned with the effects of changes in some parameters on the dynamic system. As the equilibrium point under consideration is unstable, the path after perturbations does not converge to a new equilibrium point. As we can follow the movement over time by simulation, we can see how the dynamic path is affected when there are exogenous changes in some parameters. First, we use a variable $\bar{\Delta} x(t)$ to represent the change rate of the variable $x(t)$ in percentage due to changes in the parameter value.

\subsection{The government increases the health subsidy rate}

First, we study the case that the government increases the health subsidy rate as follows: $\tau_{0}: 0.6 \Rightarrow 0.65$. The simulation result is plotted in Figure 2. As the system is unstable, we simulate the model for a short period of time. The national wealth, $A(t)=a(t) \bar{N}$, increases over time. The total labour supply and national output fall. The national debt and government's income are augmented. The industrial sector shrinks, while the health sector expands. The rate of interest rises, while the wage rate and wage income per unit of time fall. The household spends more hours on healthcare and leisure, but fewer hours on work. The price of healthcare is enhanced. The household becomes healthier, has more wealth, and consumes more goods and healthcare.

Figure 2 | The government increases the health subsidy rate

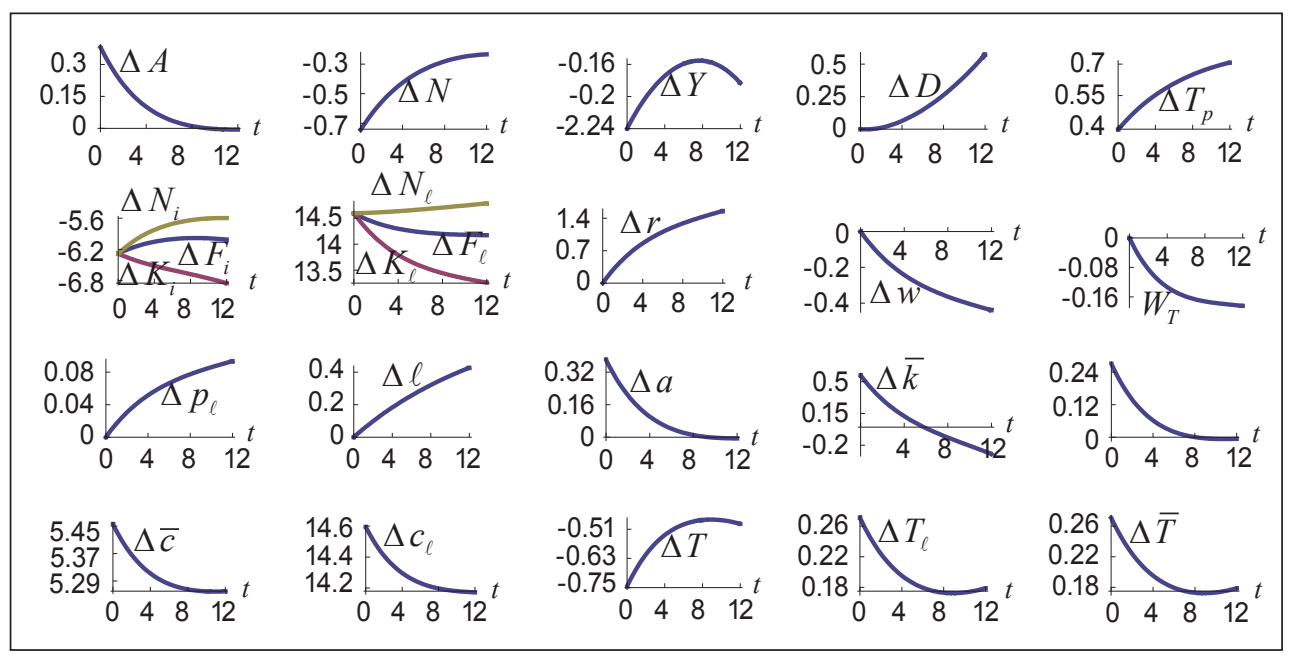

Source: author

The equilibrium point is shifted due to the change in the government policy as follows:

$\bar{\Delta} Y=0.4, \bar{\Delta} T_{p}=1, \bar{\Delta} \bar{K}=0.61, \bar{\Delta} A=0.33, \bar{\Delta} N=0.19, \bar{\Delta} D=-0.22, \bar{\Delta} K_{i}=-5.0$,

$\bar{\Delta} K_{\ell}=14, \bar{\Delta} N_{i}=-5.1, \bar{\Delta} N_{\ell}=15, \bar{\Delta} F_{i}=-5.4, \bar{\Delta} F_{\ell}=14.6, \bar{\Delta} r=1, \bar{\Delta} w=-0.28$,

$\bar{\Delta} W_{T}=0.22, \bar{\Delta} p_{\ell}=0.06, \bar{\Delta} \ell=0.83, \bar{\Delta} a=0.33, \bar{\Delta} \bar{k}=0.61, \bar{\Delta} c=0.33, \bar{\Delta} c_{\ell}=14.6$,

$\bar{\Delta} \bar{c}=5.37, \bar{\Delta} T=-0.3, \bar{\Delta} T_{\ell}=0.11, \bar{\Delta} \bar{T}=0.11$. 
We see that the conclusion from the comparative statics analysis is different from that from the short-run comparative dynamic analysis. For instance, in equilibrium, the national output is enhanced while in the short-run dynamics, the same variable falls.

\subsection{The propensity to consume healthcare is increased}

We now examine the impact of the following rise in the propensity to consume healthcare: $\Psi_{0}: 0.04 \Rightarrow 0.045$. The result is given in Figure 3. The household spends more hours on healthcare, but fewer on work and leisure activities. The health is enhanced. The total labour supply is reduced as the reduction in working hours dominates the impact by improved health. The national income and wealth fall. The national debt is increased. The government income falls initially and then rises. The interest rate rises, while the wage rate falls. The industrial sector shrinks, while the healthcare sector expands. The price of healthcare becomes higher. The household has less wealth but consumes more goods and healthcare. It is should be noted what van Zon and Muysken (2001) found a slow down in growth may be explained by a preference for health that is positively influenced by a growing income per head, or by an ageing population. Growth may virtually disappear for countries with high rates of decay of health, low productivity of the health sector, or high rates of discount.

Figure 3 | The propensity to consume healthcare is increased

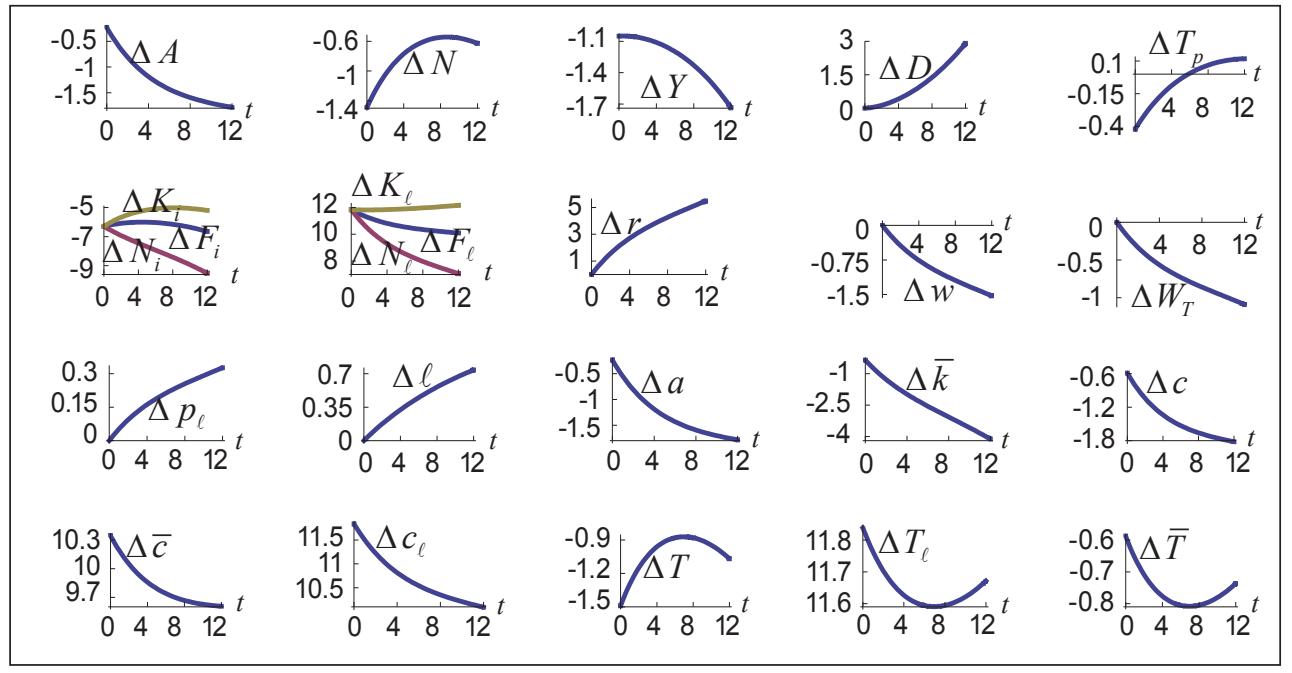

Source: author

The change in the equilibrium point is given as follows:

$$
\begin{aligned}
& \bar{\Delta} Y=0.4, \bar{\Delta} T_{p}=0.84, \bar{\Delta} \bar{K}=-0.66, \bar{\Delta} A=-1.1, \bar{\Delta} N=0.84, \bar{\Delta} D=-1.95, \\
& \bar{\Delta} K_{i}=-5.55, \bar{\Delta} K_{\ell}=9.5, \bar{\Delta} N_{i}=-3.2, \bar{\Delta} N_{\ell}=12.2, \bar{\Delta} F_{i}=-4, \bar{\Delta} F_{\ell}=11.1, \\
& \bar{\Delta} r=2.9, \bar{\Delta} w=-0.8, \bar{\Delta} W_{T}=0.03, \bar{\Delta} p_{\ell}=0.17, \bar{\Delta} \ell=1.4, \bar{\Delta} a=-1.1, \\
& \bar{\Delta} \bar{k}=-0.66, \bar{\Delta} c=-1.1, \bar{\Delta} c_{\ell}=11.1, \bar{\Delta} \bar{c}=9.8, \bar{\Delta} T=-0.01, \bar{\Delta} T_{\ell}=11.2, \\
& \bar{\Delta} \bar{T}=-1.12 .
\end{aligned}
$$




\subsection{Health productivity is enhanced}

First, we study the case where health productivity is enhanced as follows: $m_{\ell}: 0.6 \Rightarrow 0.61$. The simulation result is given in Figure 4. The health stock and total labour supply are increased. The national debt falls in association with the increases in government income. The two sectors expand. The rate of interest falls in association with rises in the wage rate. The healthcare price falls. The household has more wealth and consumes more healthcare. The household increases working hours but reduces healthcare and leisure hours.

Figure 4 | Health productive efficiency is enhanced

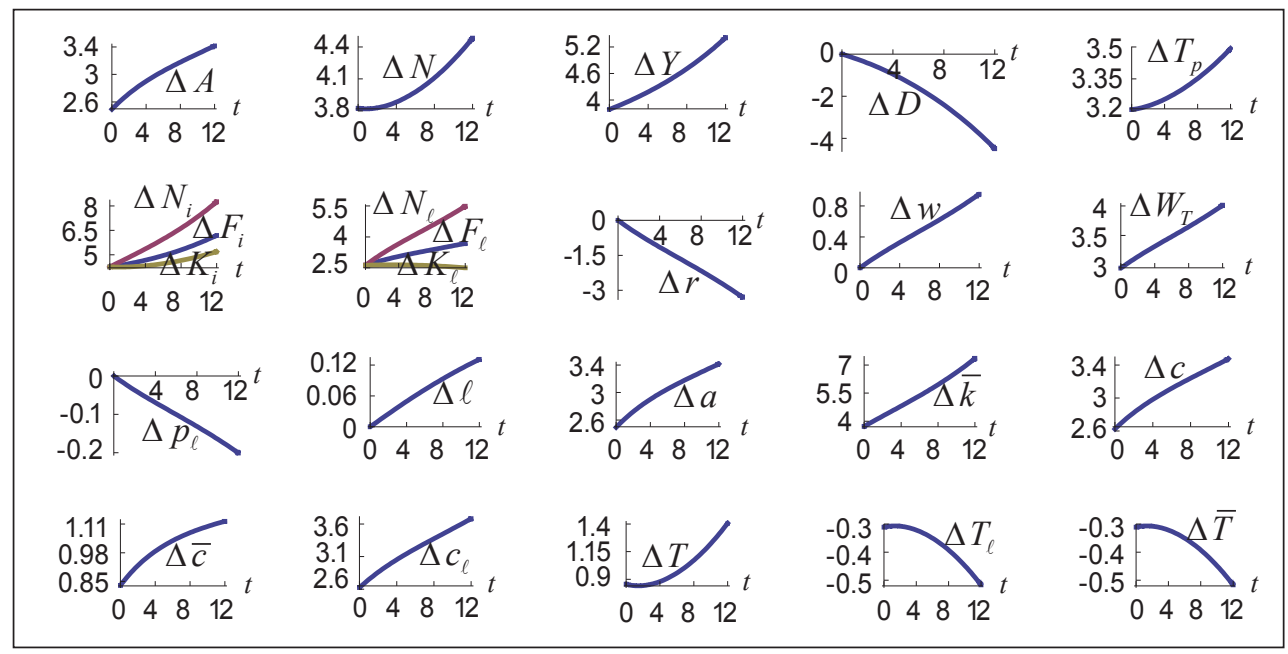

Source: author

The change in the equilibrium point is given as follows:

$\bar{\Delta} Y=3.1, \bar{\Delta} T_{p}=3.1, \bar{\Delta} \bar{K}=3.1, \bar{\Delta} A=3.1, \bar{\Delta} N=3.1, \bar{\Delta} D=3.1, \bar{\Delta} K_{i}=3.1$,

$\bar{\Delta} N_{i}=3.1, \bar{\Delta} N_{\ell}=3.1, \bar{\Delta} F_{i}=3.1, \bar{\Delta} F_{\ell}=3.1, \bar{\Delta} r=0.04, \bar{\Delta} w=-0.01$,

$\bar{\Delta} W_{T}=3.11, \bar{\Delta} p_{\ell}=0.002, \bar{\Delta} \ell=0.28, \bar{\Delta} a=3.1, \bar{\Delta} \bar{k}=3.1, \bar{\Delta} c=3.1, \bar{\Delta} c_{\ell}=3.1$,

$\bar{\Delta} \bar{c}=3.1, \bar{\Delta} T=1.18, \bar{\Delta} T_{\ell}=-0.01, \bar{\Delta} \bar{T}=0.004$.

\subsection{The total factor productivity of the industrial sector is enhanced}

We now study the case that the total factor productivity of the industrial sector is enhanced as follows: $A_{i}: 1.3 \Rightarrow 1.35$. The simulation result is plotted in Figure 5. The health stock and total labour supply are increased. The national debt falls in association with increases in government income. The two sectors' production scales expand. The two sectors employ more capital stocks. The industrial sector employs a larger labour force, while the health sector employs a smaller labour force. The interest rate initially rises and then falls. The wage rate and the healthcare price fall. The household has more wealth. The household consumes more 
goods. The household initially consumes less healthcare then consumes more. The household increases working hours but reduces healthcare and leisure hours.

Figure 5 | The total factor productivity of the industrial sector is enhanced

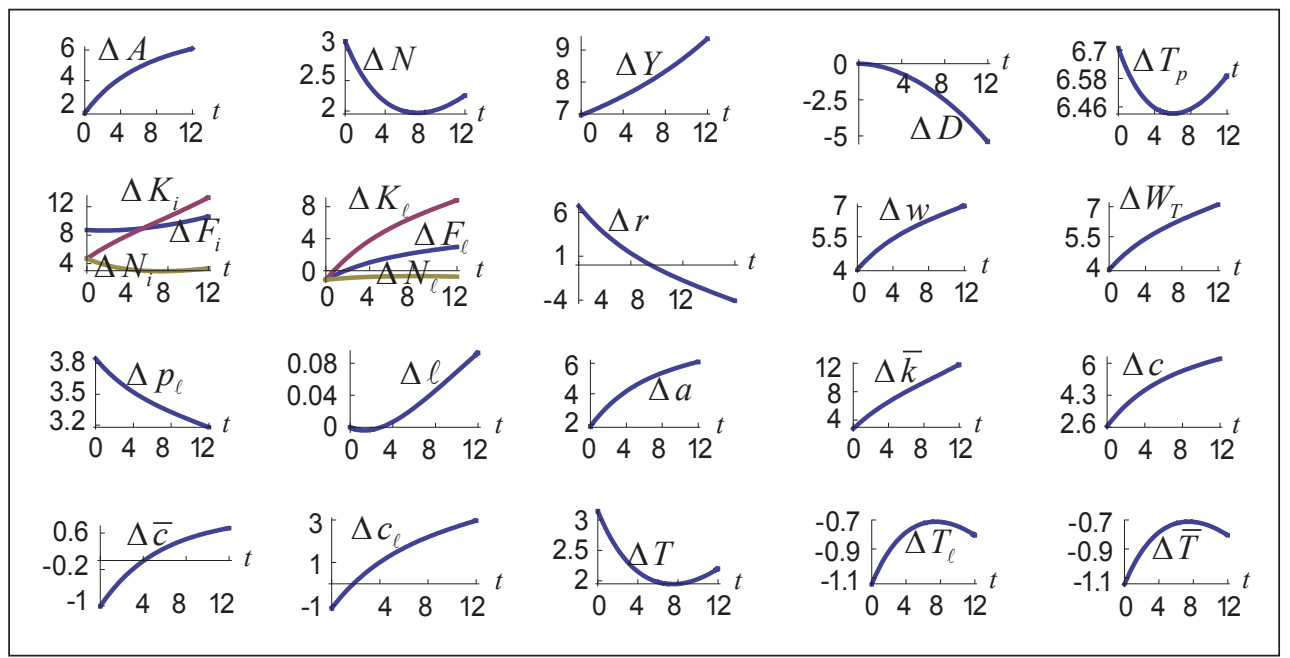

Source: author

The change in the equilibrium point is given as follows:

$$
\begin{aligned}
& \bar{\Delta} Y=6, \bar{\Delta} T_{p}=6, \bar{\Delta} \bar{K}=6, \bar{\Delta} A=6, \bar{\Delta} N=0.2, \bar{\Delta} D=6.1, \bar{\Delta} K_{i}=6, \bar{\Delta} K_{\ell}=6, \\
& \bar{\Delta} N_{i}=0.18, \bar{\Delta} N_{\ell}=0.2, \bar{\Delta} F_{i}=6, \bar{\Delta} F_{\ell}=2.5, \bar{\Delta} r=0.03, \bar{\Delta} w=5.8, \bar{\Delta} W_{T}=6, \\
& \bar{\Delta} p_{\ell}=3.44, \bar{\Delta} \ell=0.33, \bar{\Delta} a=6, \bar{\Delta} \bar{k}=6, \bar{\Delta} c=6, \bar{\Delta} c_{\ell}=2.43, \bar{\Delta} \bar{c}=0.9, \\
& \bar{\Delta} T=-0.008, \bar{\Delta} T_{\ell}=0.003, \bar{\Delta} \bar{T}=0.003 .
\end{aligned}
$$

\subsection{The efficiency of healthcare rises}

We consider an improvement in the efficiency of healthcare as follows: $A_{\ell}: 1 \Rightarrow 1.1$. The simulation result is provided in Figure 6. The health stock and total labour supply are increased. The national debt falls in association with increases in government income. The two sectors' production scales expand. The interest rate initially rises and then falls. The wage rate falls and then rises. The healthcare price falls. The household has more wealth. The household consumes more goods and more healthcare. The household increases working hours but reduces healthcare and leisure hours. 


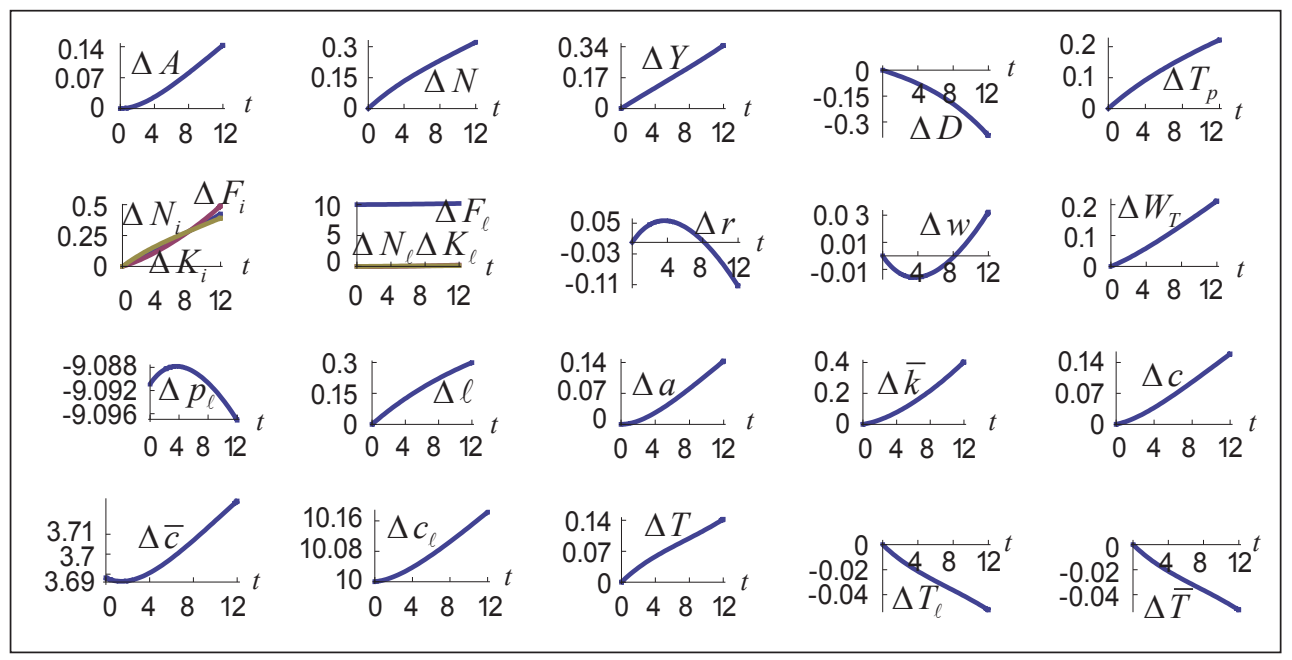

Source: author

The change in the equilibrium point is given as follows:

$$
\begin{aligned}
& \bar{\Delta} Y=0.29, \bar{\Delta} T_{p}=0.35, \bar{\Delta} \bar{K}=0.23, \bar{\Delta} A=0.34, \bar{\Delta} N=0.32, \bar{\Delta} D=0.56, \\
& \bar{\Delta} K_{i}=0.21, \bar{\Delta} K_{\ell}=0.28, \bar{\Delta} N_{i}=0.31, \bar{\Delta} N_{\ell}=0.37, \bar{\Delta} F_{i}=0.28, \bar{\Delta} F_{\ell}=10.4, \\
& \bar{\Delta} r=0.11, \bar{\Delta} w=-0.03, \bar{\Delta} W_{T}=0.33, \bar{\Delta} p_{\ell}=-9.1, \bar{\Delta} \ell=0.6, \bar{\Delta} a=0.34, \\
& \bar{\Delta} \bar{k}=0.23, \bar{\Delta} c=0.34, \bar{\Delta} c_{\ell}=10.37, \bar{\Delta} \bar{c}=3.83, \bar{\Delta} T=-0.035, \bar{\Delta} T_{\ell}=0.013, \\
& \bar{\Delta} \bar{T}=0.013 .
\end{aligned}
$$

\subsection{The tax rate on the industrial sector rises}

We now allow the tax rate on the industrial sector's output level to be increased as follows: $\tau_{i}: 0.04 \Rightarrow 0.05$. The simulation result is provided in Figure 7 . The national wealth and debt fall. The labour supply and national income initially fall and then rise. The wage rate and interest rate fall. The industrial sector initially shrinks and then expands. The household initially spends more hours on work and then fewer hours. 


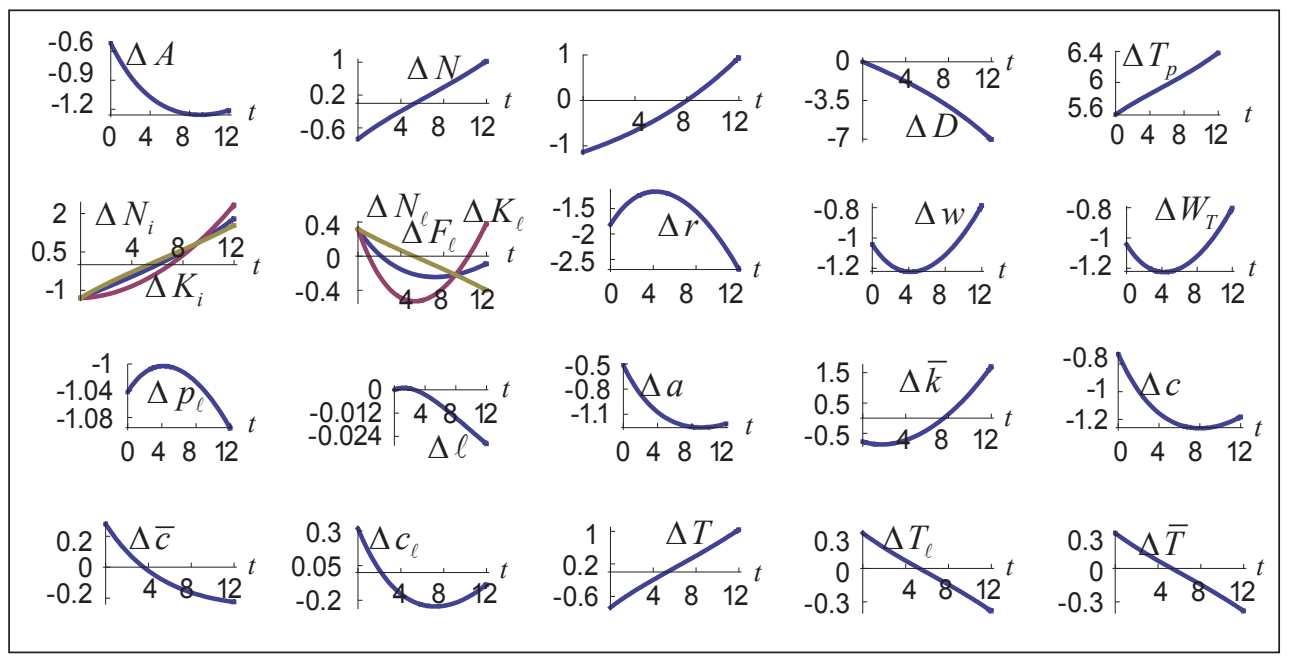

Source: author

The change in the equilibrium point is given as follows:

$\bar{\Delta} Y=-2.4, \bar{\Delta} T_{p}=5.44, \bar{\Delta} \bar{K}=-4.5, \bar{\Delta} A=-2.1, \bar{\Delta} N=-0.89, \bar{\Delta} D=2.94$, $\bar{\Delta} K_{i}=-5, \bar{\Delta} K_{\ell}=-3.5, \bar{\Delta} N_{i}=-1.3, \bar{\Delta} N_{\ell}=0.24, \bar{\Delta} F_{i}=-2.25, \bar{\Delta} F_{\ell}=-1.28$, $\bar{\Delta} r=2.64, \bar{\Delta} w=-2.28, \bar{\Delta} W_{T}=-2.35, \bar{\Delta} p_{\ell}=-0.78, \bar{\Delta} \ell=-0.11, \bar{\Delta} a=-2.05$, $\bar{\Delta} \bar{k}=-4.52, \bar{\Delta} c=-2.05, \bar{\Delta} c_{\ell}=-1.29, \bar{\Delta} \bar{c}=-0.34, \bar{\Delta} T=-0.83, \bar{\Delta} T_{\ell}=0.3$, $\bar{\Delta} \bar{T}=0.3$.

\subsection{The household augments the propensity to save}

We now deal with the case where the household increases the propensity to save as follows: $\lambda_{0}: 0.7 \Rightarrow 0.71$. The simulation result is given in Figure 8 . The national wealth and total labour supply and national output are enhanced. The national debt falls, while government income rises. The health stock is slightly changed. The household has more wealth. The consumption level initially falls and then rises. The household spends more hours on work and fewer hours on healthcare and leisure. 


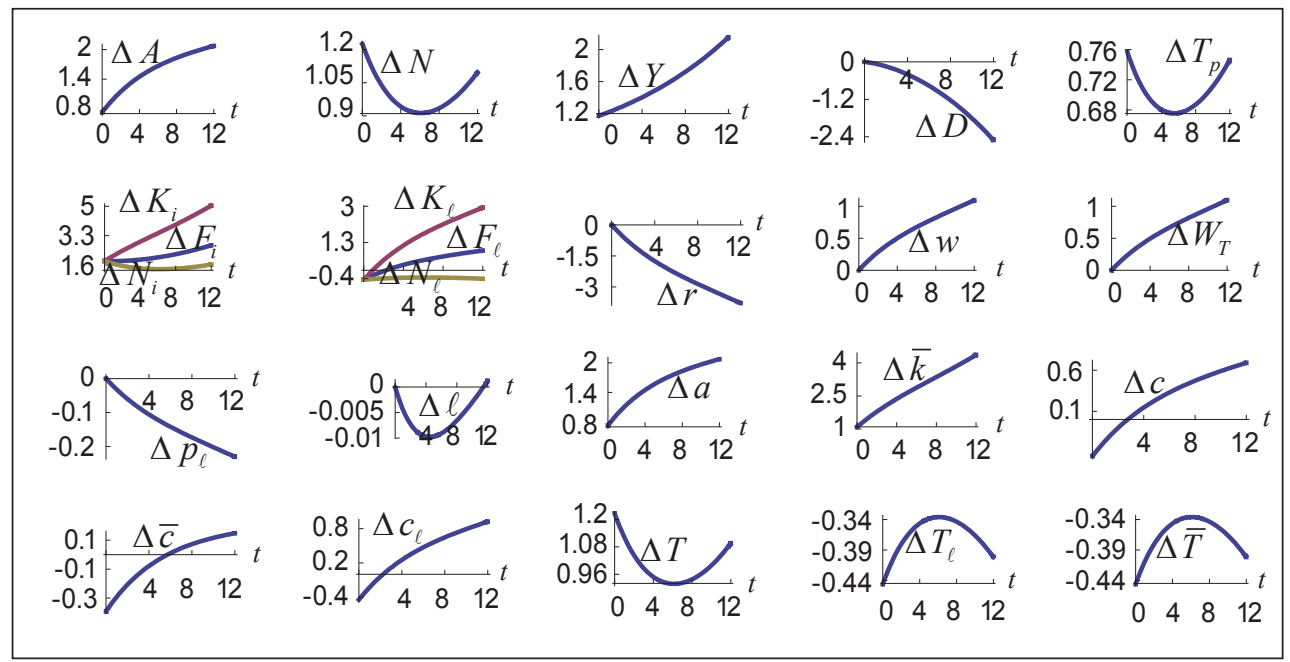

Source: author

The change in the equilibrium point is given as follows:

$$
\begin{aligned}
& \bar{\Delta} Y=0.67, \bar{\Delta} T_{p}=0.47, \bar{\Delta} \bar{K}=1.73, \bar{\Delta} A=1.94, \bar{\Delta} N=0.16, \bar{\Delta} D=2.35, \\
& \bar{\Delta} K_{i}=1.81, \bar{\Delta} K_{\ell}=1.57, \bar{\Delta} N_{i}=0.22, \bar{\Delta} N_{\ell}=-0.02, \bar{\Delta} F_{i}=0.74, \bar{\Delta} F_{\ell}=0.61, \\
& \bar{\Delta} r=-1.82, \bar{\Delta} w=0.52, \bar{\Delta} W_{T}=0.55, \bar{\Delta} p_{\ell}=-0.11, \bar{\Delta} \ell=0.05, \bar{\Delta} a=1.94, \\
& \bar{\Delta} \bar{k}=1.73, \bar{\Delta} c=0.5, \bar{\Delta} c_{\ell}=0.61, \bar{\Delta} \bar{c}=0.21, \bar{\Delta} T=0.13, \bar{\Delta} T_{\ell}=-0.05, \\
& \bar{\Delta} \bar{T}=-0.05 .
\end{aligned}
$$

\section{Conclusions}

The paper studies the dynamic interdependence between wealth accumulation and health accumulation with government health policy. We were concerned with government debts and health policy in a dynamic general equilibrium analytical framework. We built the general equilibrium growth model with health and debt and found three nonlinear differential equations to describe the movement of the economic system. We simulated the model and showed how the economic system reacts to different exogenous changes in the short term and in the equilibrium point and provided some insights into complicated interactions between variables. For instance, if the government increases the subsidy rate for health, we have the following effects in the short term: the national wealth increases over time; the total labour supply and national output fall; the government debt and income are augmented; the industrial sector shrinks, while the health sector expands; the rate of interest rises, while the wage rate falls; the household spends more hours on healthcare and leisure, but fewer hours on work; the price of healthcare is increased; the household becomes healthier, has more wealth, and consumes more goods and healthcare. It should be noted that we can extend 
the model in different ways. As our model was developed in a general equilibrium framework and we provided a computational procedure to calculate all the variables, we can easily analyse the effects of the changes in other parameters. As it is influenced by well-known economic models and each of these models has a large amount of literature regarding generalisations and extensions, we can further develop our model on the basis of the literature.

\section{Appendix: Proving the Lemma}

Equations (2) and (3) imply

$$
z \equiv \frac{r+\delta_{k}}{w}=\frac{N_{q}}{\bar{\beta}_{q} K_{q}}
$$

where

$$
\bar{\beta}_{q} \equiv \frac{\beta_{q}}{\alpha_{q}}, q=i, \ell .
$$

From (A1) and (3) we obtain

$$
r(z, D)=\alpha z^{\beta_{i}}-\delta_{k},
$$

where

$$
\alpha \equiv \bar{\tau}_{i} \alpha_{i} A_{i} \bar{\beta}_{i}^{\beta_{i}} .
$$

From (A1) and (A3), we obtain

$$
w(z, D)=\frac{r+\delta_{k}}{z} .
$$

With (3) and (A1), we have

$$
p_{\ell}(z, D)=\frac{\bar{\beta}_{\ell}^{\alpha_{\ell}} w z^{\alpha_{\ell}}}{\bar{\tau}_{\ell} \beta_{\ell} A_{\ell}} .
$$

With (10), we have

$$
\bar{y}=\tilde{R} a+W,
$$

where $W \equiv T_{0} \tilde{w}$. Insert (12) in (8)

$$
T=T_{0}-\left(\sigma+\psi_{T}\right) \frac{\bar{y}}{\tilde{w}} .
$$

Insert (A5) in (A6)

$$
T=r_{0}-R_{0} a,
$$

where

$$
r_{0} \equiv\left(1-\sigma-\psi_{T}\right) T_{0}, \quad R_{0} \equiv\left(\sigma+\psi_{T}\right) \frac{\tilde{R}}{\tilde{w}} .
$$


Substitute (A7) into (1)

$$
N=\bar{r}-\bar{R} a,
$$

where

$$
\bar{r} \equiv r_{0} \bar{\ell} \bar{N}, \bar{R} \equiv R_{0} \bar{\ell} \bar{N} .
$$

From (13) and (19) we have

$$
N_{\ell}=\frac{\psi_{\ell} \bar{y} \bar{N}}{f_{\ell} \bar{p}_{\ell}},
$$

where we also use (3) and (2) and

From (16), (3) and (2), we have

$$
f_{\ell} \equiv \frac{F_{\ell}}{N_{\ell}}=\frac{A_{\ell}}{\left(\bar{\beta}_{\ell} z\right)^{\alpha_{\ell}}} .
$$

$$
T_{p}=\tau_{i} f_{i} N_{i}+\tau_{\ell} p_{\ell} f_{\ell} N_{\ell}+I_{c} \bar{N},
$$

where

Insert (22) in (A10)

$$
f_{i} \equiv \frac{F_{i}}{N_{i}}=\frac{A_{i}}{\left(\bar{\beta}_{i} z\right)^{\alpha_{i}}}, \quad I_{c} \equiv \tau_{a} r a+\tau_{c} c+\tau_{w} \bar{\ell} T w .
$$

$$
T_{p}=(\bar{r}-\bar{R} a) \tau_{i} f_{i}+\bar{p} \bar{y}+I_{c} \bar{N},
$$

in which we also use (A8) and (A9) and

$$
\bar{p} \equiv\left(\tau_{\ell} p_{\ell} f_{\ell}-\tau_{i} f_{i}\right) \frac{\psi_{\ell} \bar{N}}{f_{\ell} \bar{p}_{\ell}} .
$$

Insert (A7) and (12) into the definition of $I_{c}$

$$
I_{c}=\left(\tau_{a} r-\tau_{w} \bar{\ell} w R_{0}\right) a+\tau_{c} \xi \bar{y}+\tau_{w} \bar{\ell} w r_{0} .
$$

Insert (A12) in (A11)

$$
T_{p}=I_{0}+I_{a} a,
$$

where we use (A5) and

$$
\begin{aligned}
& I_{0} \equiv \bar{r} \tau_{i} f_{i}+\left(\bar{p}+\tau_{c} \xi \bar{N}\right) W+\tau_{w} \bar{\ell} w r_{0} \bar{N} \\
& I_{a} \equiv\left(\bar{p}+\tau_{c} \xi \bar{N}\right) R+\tau_{a} r \bar{N}-\tau_{w} \bar{\ell} w R_{0} \bar{N}-\bar{R} \tau_{i} f_{i} .
\end{aligned}
$$


From (22) and (23), we have

$$
\frac{N_{i}}{\bar{\beta}_{i}}+\frac{N_{\ell}}{\bar{\beta}_{\ell}}=z \bar{k} \bar{N}
$$

where we also use (A1). With (22), we have

$$
\frac{N}{\bar{\beta}_{i}}+\hat{\beta} N_{\ell}=z \bar{k} \bar{N}
$$

where

$$
\hat{\beta} \equiv \frac{1}{\bar{\beta}_{\ell}}-\frac{1}{\bar{\beta}_{i}}
$$

Insert (A8) and (A9) in (A15)

$$
a(z, D, \ell)=\left(\frac{\bar{r}}{\bar{\beta}_{i}}+\frac{\hat{\beta} \psi_{\ell} \bar{N} W}{f_{\ell} \bar{p}_{\ell}}+z D\right)\left(z \bar{N}+\frac{\bar{R}}{\bar{\beta}_{i}}-\frac{\hat{\beta} \psi_{\ell} \bar{N} \tilde{R}}{f_{\ell} \bar{p}_{\ell}}\right)^{-1},
$$

where we use (A5), $a=d+\bar{k}$, and $D=d \bar{N}$.

We solve all the variables as functions of $z, \quad \ell$ and $D$ at any point in time as follows: $r$ by (A2) $\rightarrow w$ with (A3) $\rightarrow p_{\ell}$ by (A4) $\rightarrow a$ with (A16) $\rightarrow \bar{y}$ from (A5) $\rightarrow d=D / \bar{N}$ $\rightarrow \bar{k}=a-d \rightarrow s, \hat{T}, \bar{T}, c$, and $c_{\ell}$ by (13) $\rightarrow T$ with (9) $\rightarrow N$ by (1) $\rightarrow N_{\ell}$ from (A9) $\rightarrow N_{i}=N-N_{\ell} \rightarrow K_{i}, \quad K_{\ell}$ by (A1) $\rightarrow K$ by (22) $\rightarrow F_{q}$ by (2) $\rightarrow \bar{c}$ from (4) $\rightarrow T_{p}$ by (A13). From this procedure, (14), (18), and (21), we get

$$
\begin{gathered}
\dot{a}=\Lambda_{0}(z, \ell, D) \equiv s-a, \\
\dot{D}=\Lambda_{D}(z, \ell, D) \equiv r D+\tilde{\tau} p_{\ell} \bar{N}-T_{p}, \\
\dot{\ell}=\Lambda_{\ell}(z, \ell, D) \equiv \frac{\bar{v}_{\ell} \bar{c}^{\bar{a}_{\ell}}}{\ell^{\bar{\pi}_{\ell}}}+\frac{\bar{v}_{c} c^{\bar{a}_{c}}}{\ell^{\bar{\pi}_{c}}}+\frac{\bar{v}_{T} \bar{T}^{\bar{a}_{T}}}{\ell^{\bar{\pi}_{T}}}-\delta_{\ell} \ell .
\end{gathered}
$$

Take the derivatives of (A16) with respect to $t$

$$
\dot{a}=\frac{\partial a}{\partial z} \dot{z}+\Lambda_{\ell} \frac{\partial a}{\partial \ell}+\Lambda_{D} \frac{\partial \Lambda}{\partial D}
$$

where we also apply (A18). From (A17) and (A19), we have

$$
\dot{z}=\Lambda_{z}(z, \ell, D) \equiv\left(\Lambda_{0}-\Lambda_{\ell} \frac{\partial a}{\partial \ell}-\Lambda_{D} \frac{\partial a}{\partial D}\right)\left(\frac{\partial a}{\partial z}\right)^{-1} .
$$

We have three differential equations (A19) and (A20) with three variables. We have the other variables with the procedure in the Lemma. 


\section{References}

Abel, A., Bernanke, B. S. and Croushore, D. (2007). Macroeconomics. New Jersey: Prentice Hall.

Acemoglu, D. and Johanson, S. (2007). Disease and Development: The Effect of Life Expectancy. Journal of Political Economy, 115(6), pp. 925-985, https://doi.org/ 10.3386/w12269

Baltagi, B. H. and Moscone, F. (2010). Health Care Expenditure and Income in the OECD Reconsidered: Evidence from Panel Data. Economic Modelling, 27(4), pp. 804-811, https://doi.org/10.1016/j.econmod.2009.12.001

Bedir, S. (2016). Health Care Expenditure and Economic Growth in Developing Countries. Advances in Economics and Business, 4(2), pp. 76-86, https://doi.org/10.13189/aeb.2016.040202

Bloom, D. E. and Canning, D. (2003). The Health and Poverty of Nations: From Theory to Practice. Journal of Human Development, 4(1), pp. 47-71, https://doi.org/10.1080/1464988032000051487

Bloom, D. E., Canning, D. and Sevilla, J. (2004). The Effect of Health on Economic Growth: A Production Function Approach. World Development, 32(1), pp. 1-13, https://doi.org/10.1016/j.worlddev.2003.07.002

Burmeister, E. and Dobell, A. R. (1970). Mathematical Theories of Economic Growth. London: Collier Macmillan Publishers.

Chen, W., Clarke, J. A. and Roy, N. (2014). Health and Wealth: Short Panel Causality Tests for Developing Countries. Journal of International Trade \& Economic Development, 23(6), pp. 755-784, https://doi.org/10.1080/09638199.2013.783093

Diamond, P. (1965). National Debt in a Neoclassical Growth Model. American Economic Review, 55(5), pp. 1126-1150.

Eggoh, J., Houeninvo, H. and Sossou, G. A. Hilaire, E. J. and Gilles, A. S. (2015). Education, Health and Economic Growth in African Countries. Journal of Economic Development, 40(1), pp. 93-111.

Ehrlich, I. and Chuma, H. (1990). A Model of the Demand for Longevity and the Value of Life Extension. Journal of Political Economy, 98(4), pp. 761-782, https://doi.org/10.1086/261705

Forster, B. A. (1989). Optimal Health Investment Strategies. Bulletin of Economic Research, 41(1), pp. 45-57, https://doi.org/10.1111/j.1467-8586.1989.tb00277.x

Grossman, M. (1972). On the Concept of Health Capital and the Demand of Health. Journal of Political Economy, 80(2), pp. 223-255, https://doi.org/10.1086/259880

Johansson, P. O. and Löfgren, K. G. (1995). Wealth from Optimal Health. Journal of Health Economics, 14(1), pp. 65-79, https://doi.org/10.1016/0167-6296(94)00037-5

Miles, D. and Scott, A. (2005). Macroeconomics - Understanding the Wealth of Nations. Chichester: John Wiley \& Sons, Ltd.

Murray, C. J. L., Govindaraj, R. and Musgrove, P. (1994). National Health Expenditures: A Global Analysis. Bulletin of the World Health Organization, 72(4), pp. 623-637.

Newhouse, J. P. (1977). Medical Care Expenditure: A Cross-National Survey. Journal of Human Resources, 12, pp. 115-125, https://doi.org/10.2307/145602

Parkin, D., McGuire, A. and Yle, B. (1987). Aggregate Health Care Expenditures and National Income: Is Health Care a Luxury Good? Journal of Health Economics, 6(2), pp. 109-127, https://doi.org/10.1016/0167-6296(87)90002-6

Posnett, J. and Hitiris, T. (1992). The Determinants and Effects of Health Expenditure in Developed Countries. Journal of Health Economics, 11(2), pp. 173-181, https://doi.org/10.1016/0167-6296(92)90033-W 
Schultz, P. (1999). Health and Schooling Investments in Africa. Journal of Economic Perspectives, 13(3), pp. 67-88.

Solow, R. (2000). Growth Theory - An Exposition (2nd ed.). New York: Oxford University Press.

Strauss, J. and Thomas, D. (1998). Health, Nutrition, and Economic Development. Journal of Economic Literature, 36(2), pp. 766-817.

Tobing, E. (2011). Public Health Spending, Tax Reform, and Long-Run Growth. Economics Letters, 112(1), pp. 119-121.

Van Zon, A. and Muysken, J. (2001). Health and Endogenous Growth. Journal of Health Economic, 20, pp. 169-185, https://doi.org/10.1016/S0167-6296(00)00072-2

Well, D. N. (2007). Accounting for the Effect of Health on Education Growth. The Quarterly Journal of Economics, 122(3), pp. 1265-1306, https://doi.org/10.1162/qjec.122.3.1265

Yavuz, N. C., Yilanci, V. and Ozturk, Z. A. (2011). Is Health Care a Luxury or a Necessity or Both? Evidence from Turkey. European Journal of Health Economics, 14(1), pp. 5-10, https://doi.org/10.1007/s10198-011-0339-6

Zhang, W. B. (1993). Woman's Labor Participation and Economic Growth - Creativity, Knowledge Utilization and Family Preference. Economics Letters, 42(1), pp. 105-110, https://doi.org/10.1016/0165-1765(93)90181-B

Zhang, W. B. (2005). Economic Growth Theory. London: Ashgate.

Zhang, W. B. (2016). Public Debt and Economic Growth in Uzawa's Two-Sector Model with Public Goods. International Journal of Economic Sciences, 5(4), pp. 51-72, https://doi.org/10.20472/ES.2016.5.4.004

Zhang, W. B. (2018). Dynamic Interactions Between Health, Human Capital and Wealth. Academicus International Scientific Journal, 17(January), pp. 122-145. 